\title{
Combinatorial Perron Parameters for Trees
}

\author{
Enide Andrade* \\ Lorenzo Ciardo ${ }^{\dagger}$ \\ Geir Dahl ${ }^{\ddagger}$
}

Dec. 26,2018

Published in Linear Algebra Appl. (2019)

\begin{abstract}
The notion of combinatorial Perron value was introduced in [2]. We continue the study of this parameter and also introduce a new parameter $\pi_{e}(M)$ which gives a new lower bound on the spectral radius of the bottleneck matrix $M$ of a rooted tree. We prove a bound on the approximation error for $\pi_{e}(M)$. Several properties of these two parameters are shown. These ideas are motivated by the concept of algebraic connectivity. A certain extension property for the combinatorial Perron value is shown and it allows us to define a new center concept for caterpillars. We also compare computationally this new center to the so-called characteristic set, i.e., the center obtained from algebraic connectivity.
\end{abstract}

Key words. Perron value; bottleneck matrix; tree; Laplacian matrix; majorization

AMS subject classifications. 15A18; $05 \mathrm{C} 05$

*Center for Research and Development in Mathematics and Applications, Department of Mathematics, University of Aveiro, Portugal. enide@ua.pt

${ }^{\dagger}$ Department of Mathematics, University of Oslo, Norway. lorenzci@math.uio.no

${ }_{\ddagger}^{\ddagger}$ Department of Mathematics, University of Oslo, Norway. geird@math.uio.no. Corresponding author. 


\section{Introduction}

The purpose of this paper is to investigate combinatorial parameters associated with bottleneck matrices of trees. A new such parameter will be introduced. Bottleneck matrices arise naturally from the Laplacian matrix of a tree in connection with the notion of algebraic connectivity, as explained below. The main goals of this paper are:

1. For a rooted tree with corresponding bottleneck matrix $M$ we introduce a new parameter $\pi_{e}(M)$ which is a (usually good) lower bound on the spectral radius $\rho(M)$. We show different properties of $\pi_{e}(M)$, including a result for caterpillars which involves the notion of majorization.

2. We study the combinatorial Perron value $\rho_{c}(M)$, introduced in [2], and prove several properties. These properties are used to define a new center concept for caterpillars, which then is compared to the center based on Perron values.

3. We also give exact values for $\pi_{e}(M), \pi_{d}(M)$ (also introduced in [2]) and $\rho_{c}(M)$ for certain trees.

In the remaining part of this introduction we give some preliminaries about algebraic connectivity, bottleneck matrices and related concepts. Let $G=$ $(V, E)$ be an undirected simple graph and $L(G)$ its Laplacian matrix, i.e., $L(G)=D-A$ where $A$ is the adjacency matrix and $D$ the diagonal matrix of vertex degrees. $L(G)$ is positive semidefinite and singular, and we order its eigenvalues as follows: $\mu_{1} \geq \mu_{2} \geq \cdots \geq \mu_{n}=0$. The multiplicity of the eigenvalue 0 of $L(G)$ equals the number of connected components of $G$. Thus, for connected graphs the second smallest eigenvalue $\mu_{n-1}$ is positive. Fiedler [4] studied this eigenvalue and called $\mu_{n-1}$ the algebraic connectivity of $G$, denoted by $a(G)$. Since then, $a(G)$ is considered to be a very important parameter, and it is used as a measure of the connectivity of a graph $G$. Moreover, if $T$ is a tree, $a(T)$ is closely related to the Perron value (spectral radius) of so-called bottleneck matrices of subtrees of $T$, as explained below.

An important tool in connection with algebraic connectivity is the Fiedler's Monotonicity Theorem. This theorem describes the structure of the eigenvectors associated to $a(G)$, and it has led to a large number of papers about ordering graphs by this invariant, mainly graphs such as trees and graphs with few cycles, see e.g. [1] for a survey on the significant results concerning these topics. 
If $z=\left(z_{1}, z_{2}, \ldots, z_{n}\right)$ is an eigenvector corresponding to $a(G)$, here called a Fiedler vector, we may label the vertex $v_{i}$ (the order induced by the Laplacian matrix) with $z_{i}$. Fiedler showed that this labeling, called a characteristic valuation, gives information about the graph. In this paper, the results will be presented for a general tree $T$.

Theorem 1.1. (Fiedler's Monotonicity Theorem) If $z$ is an eigenvector corresponding to a $(T)$ (now called Fiedler vector), then exactly one of the following two cases occurs:

(1) Some entry of $z$ is 0 . In this case, the subgraph induced by the vertices corresponding to zeros in $z$ is connected. Moreover, there is a unique vertex $v_{k}$ such that $z_{k}=0$ and $v_{k}$ is adjacent to a vertex $v_{i}$ where $z_{i} \neq 0$.

(2) No entry of $z$ is 0 . In this case, there is a unique pair of vertices $v_{i}$ and $v_{j}$ such that $v_{i}$ is adjacent to $v_{j}$ with $z_{i}>0$ and $z_{j}<0$. Furthermore, the entries of $z$ are increasing along any path in $T$ which starts at $v_{i}$ and does not contain $v_{j}$, and the entries of $z$ are decreasing along any path in $T$ which starts at $v_{j}$ and does not contain $v_{i}$.

Based on this theorem Merris [10] classified trees into two types. Trees that satisfy Case 1 of the previous theorem are called Type I trees. In this case, the vertex $v_{k}$ is called the characteristic vertex of $T$. Trees that satisfy Case 2 of the previous theorem are called Type II trees. In this case, the vertices $v_{i}$ and $v_{j}$ are called the characteristic vertices of $T$. The characteristic set of a tree is the set of its characteristic vertices. Note that the characteristic set of $T$ is independent of the eigenvector used as the characteristic valuation.

Algebraic connectivity and Fiedler vectors for trees have been studied in several papers, see, e.g., $[5,6,7,8,10]$; see also the book [12]. These results lead to the study of the inverses of submatrices of $L(T)$. Let $v$ be a vertex of $T$ and let $L_{v}$ be the principal submatrix of $L(T)$ obtained by deleting the row and column corresponding to $v$. Then $L_{v}$ is invertible and its inverse $L_{v}^{-1}$ is the direct sum of blocks corresponding to each of the branches of $T$ at $v$. We call each such block a bottleneck matrix (of that branch). Thus, a bottleneck matrix $M$ corresponds to a rooted tree $B$ with root $r$ which is the unique vertex in $B$ that is adjacent to $v$ in $T$. One can show $([8,12])$ that the $(i, j)$ 'th entry of $M$ is the number of vertices (in $B$ ) that lie simultaneously in the path between $r$ and $i$ and in the path between $r$ and $j$.

Recall from the Perron-Frobenius theory for nonnegative matrices that the spectral radius of a positive matrix $M$ is necessarily an eigenvalue of 
that matrix, called the Perron value and denoted by $\rho(M)$. Hence it follows that the spectral radius of $L_{v}^{-1}$ is equal to the Perron value of the bottleneck matrix (matrices) for a branch at $v$ whose Perron value is largest. A branch at a vertex $k$ whose bottleneck matrix has the largest Perron value is a Perron branch at $v$.

It is then possible to characterize, via bottleneck matrices, Type I and Type II trees (see [8]). In fact, $T$ is a Type I tree with characteristic vertex $v$ if and only if $T$ has two or more Perron branches at $v$. In this case, the algebraic connectivity is the inverse of the Perron value of the bottleneck matrix of any of its Perron branches. Moreover, $T$ is a Type II tree with (adjacent) characteristic vertices $v_{i}$ and $v_{j}$ if and only if there exists $\gamma, 0<\gamma<1$, such that $\rho\left(M_{1}-\gamma J\right)=\rho\left(M_{2}-(1-\gamma) J\right)$, where $J$ is the all ones matrix and $M_{1}$ is the bottleneck matrix for the branch at $v_{j}$ containing $v_{i}$ and $M_{2}$ is the bottleneck matrix for the branch at $v_{i}$ containing $v_{j}$. Then the algebraic connectivity can be obtained by

$$
a(T)=\frac{1}{\rho\left(M_{1}-\gamma J\right)}=\frac{1}{\rho\left(M_{2}-(1-\gamma) J\right)} .
$$

Therefore, we can find the characteristic set by computing the spectral radius of bottleneck matrices.

In [2] one introduced a parameter called the combinatorial Perron value for a tree $T$, denoted by $\rho_{c}(T)$. Moreover, the related parameter $\pi_{d}(T)$ was also introduced. A motivation for the concept of combinatorial Perron value was that the vector of distances from the root (in branches) has some similarity to the corresponding Perron vector, and the Fiedler vector of an underlying tree. These two parameters were shown in [2] to provide very good lower bounds on the Perron value of the associated bottleneck matrix, and several theoretical properties were established.

Notation: We usually let the vertex set of a rooted tree be labelled $1,2, \ldots, n$, where $r=1$ is the root. Two special rooted trees are the star and the path. We denote by $\mathcal{S}_{n}$ the star with $n$ vertices having the central vertex as root; therefore, we label the central vertex as 1 and the remaining vertices as $2,3, \ldots, n$ in no specific order. Also, we denote by $\mathcal{P}_{n}$ the path with $n$ vertices having one of the endpoints as root; its vertices are labeled from 1 (the root) to $n$ (the other endpoint) in the natural way. The all ones vector (of suitable dimension) is denoted by $e$. The transpose of a matrix $A$ is denoted by $A^{T}$, and its trace is denoted by $\operatorname{Tr} A$. The spectral norm of a matrix, and the Euclidean norm of a vector, are denoted by $\|\cdot\|$. Also, for a 
square matrix $A=\left[a_{i j}\right]$, we use the matrix norms $\|A\|_{\infty}=\max _{i} \sum_{j}\left|a_{i j}\right|$ and $\|A\|_{1}=\max _{j} \sum_{i}\left|a_{i j}\right|$. Vectors are treated as column vectors, and, whenever convenient, these are identified with corresponding $n$-tuples.

\section{A new combinatorial Perron parameter}

In this section we recall some concepts from [2], and introduce a new combinatorial parameter associated with bottleneck matrices.

Let the pair $(T, r)$ denote a rooted tree, consisting of a tree $T$ and a specified vertex $r$ (in $T$ ) called the root. Let $n$ be the number of vertices of $T$, and let, for each vertex $j, P_{j}$ denote the (unique) $r j$-path in $T$. The path matrix $N$ (of $T$ ) is the $(0,1)$-matrix of size $n \times n$ whose rows and columns correspond to vertices in $T$, and where column $j$ of $N$ is the incidence vector of the path $P_{j}$ for $j \leq n$, i.e., it contains ones in rows corresponding to vertices in $P_{j}$, and zeros elsewhere. With suitable ordering of the vertices, the path matrix $N$ is upper triangular with ones in the first row and on the diagonal. Its column sum vector is the distance vector $d=\left(d_{1}, d_{2}, \ldots, d_{n}\right)$, where $d_{j}$ is the number of vertices in $P_{j}$ and represents the distance of vertex $j$ to the root. The bottleneck matrix $M$ and the path matrix $N$ are related by $M=N^{T} N$. For instance, for the star $\mathcal{S}_{n}$ (with, as said above, the central vertex as its root, and labeled 1) then its bottleneck matrix is the $n \times n$ matrix $M=\left[m_{i j}\right]$ where $m_{22}=m_{33}=\cdots=m_{n n}=2$ and all other entries are 1 .

The following two parameters were introduced in [2]:

$$
\rho_{c}(M)=\frac{\|N d\|^{2}}{\|d\|^{2}}=\frac{d^{T} M d}{d^{T} d}, \text { and } \quad \pi_{d}(M)=\frac{\|M d\|}{\|d\|} .
$$

Both of these provide lower bounds on the Perron value $\rho(M)$ of the bottleneck matrix $M$. (The notation for $\pi_{d}(M)$ was $\pi(N)$, or $\pi(T)$, in [2]). An alternative expression for $\rho_{c}(M)$ is

$$
\rho_{c}(M)=\frac{\sum_{i} \sigma_{i}^{2}}{\sum_{i} d_{i}^{2}}
$$

where $\sigma_{i}=\sum_{j: j \preceq i} d_{j}$ and $j \preceq i$ means that $j$ is below $i$ in the sense that the path $P_{i}$ is contained in the path $P_{j}$. We shall refer to $\sigma_{i}$ as the weight of vertex $i$. 
Theorem 2.1. ([2]) Let $T$ be a rooted tree (as above), and $M$ its bottleneck matrix. Then

$$
\rho_{c}(M) \leq \pi_{d}(M) \leq \rho(M) \leq\|M\|_{1} .
$$

Now, we introduce a new related parameter, defined by

$$
\pi_{e}(M)=\|M e\| /\|e\|=\left[\frac{1}{n} \sum_{i}\left(\sum_{j}\left|P_{i} \cap P_{j}\right|\right)^{2}\right]^{1 / 2} .
$$

We also write $\pi_{e}(T)$ for the same expression. Note that $\sum_{j}\left|P_{i} \cap P_{j}\right|$ is the $i$ 'th row sum in $M$, and that $\pi_{e}$ is given by a combinatorial expression (although involving a square root). We will refer to all of these parameters, $\rho_{c}(M)$, $\pi_{d}(M)$ and $\pi_{e}(M)$, as combinatorial Perron parameters.

We can relate this new parameter $\pi_{e}(M)$ to the Perron value by using the connection to the operator norm of the bottleneck matrix. We have that $\rho(M)=\|M\|=\max _{x \neq 0}\|M x\| /\|x\|$. If we here let $x=d$, the distance vector, we obtain $\|M d\| /\|d\|=\pi_{d}(M)$. An even simpler choice is to let $x=e$, the all ones vector, which gives $\|M e\| /\|e\|=\pi_{e}(M)$. Both of these numbers are therefore lower bounds on $\rho(M)$. The expression for $\pi_{e}(M)$ is simpler than those of $\rho_{c}(M)$ and $\pi_{d}(M)$, in the sense that the denominator is just $\sqrt{n}$. This has the consequence that some questions are easier to study for $\pi_{e}$, and, as we shall see, it has some nice properties.

Extensive computational experiments suggest that the relative error of these parameters as approximations to the Perron value is typically around $3-5 \%$ for random trees up to 100 vertices. It is an interesting, but difficult, question to prove bounds on the mentioned approximation errors. The following is one result in this direction, and it concerns $\pi_{e}(M)$.

Let $M$ be a symmetric, nonnegative matrix of order $n$. We are mainly interested in the case when $M$ is the bottleneck matrix of a rooted tree $T$. Let $M^{2}=\left[p_{i j}\right]$. Let $\beta \geq 0$ and consider the inequality

$$
\sum_{i, j=1}^{n} p_{i j} \geq \beta n \sum_{i=1}^{n} p_{i i} .
$$

If (3) holds, we say that $M$ is $\beta$-close. This is a condition on the inner products of (pairs of) rows in $M$, which expresses, roughly speaking, a comparison between the average inner product of pairs of rows in $M$ and the average inner product of each row with itself; the gap is measured by $\beta$. With this notation we have the following result. 
Theorem 2.2. Assume that $M$ is $\beta$-close. Then

$$
\beta^{1 / 2} \rho(M) \leq \pi_{e}(M) \leq \rho(M) .
$$

Proof. The right-most inequality holds in general, as explained above, and does not require the extra-condition (3). Since $M$ is symmetric,

$$
\pi_{e}(M)=\|M e\| /\|e\|=\left((1 / n) e^{T} M^{2} e\right)^{1 / 2}
$$

which is the square root of the sum of all entries of $M^{2}$ divided by $n$. Recall that the Frobenius norm of $M$ is given by $\|M\|_{F}=\sqrt{\operatorname{Tr}\left(M^{T} M\right)}=$ $\sqrt{\operatorname{Tr}\left(M^{2}\right)}$, and, since this norm is a consistent matrix norm, $\rho(M) \leq\|M\|_{F}$. Now, $\operatorname{Tr}\left(M^{2}\right)=\sum_{i} p_{i i}$, so by $(3)$

$$
\begin{aligned}
\pi_{e}(M) & =\left(\frac{1}{n} \sum_{i, j=1}^{n} p_{i j}\right)^{1 / 2} \\
& \geq\left(\frac{1}{n} \beta n \sum_{i=1}^{n} p_{i i}\right)^{1 / 2} \\
& =\beta^{1 / 2}\|M\|_{F} \\
& \geq \beta^{1 / 2} \rho(M) .
\end{aligned}
$$

Therefore, if $M$ is $\beta$-close for some $\beta$ close to 1 , then $\pi_{e}(M)$ is necessarily a good approximation to the Perron value $\rho(M)$. From experiments we see that random trees often satisfy this condition. Clearly, the optimal $\beta$ for a given tree $T$ is given by

$$
\beta^{*}(T):=\frac{\sum_{i, j} p_{i j}}{n \sum_{i} p_{i i}} .
$$

Consider given integers $k \geq 1$ and $z \geq 0$, and the generalized star $G_{k, z}$ which is the tree obtained by taking $k$ paths of length $z+1$ and identifying their roots. The total number of vertices in $G_{k, z}$ is then $1+k z$. Computations show that the exact value of $\beta^{*}$ for the generalized star $G_{k, z}$ is

$$
\frac{30+30 k^{3} z^{3}+10 k^{2} z^{2}\left(10+3 z+2 z^{2}\right)+k z\left(101+35 z+30 z^{2}+10 z^{3}+4 z^{4}\right)}{5\left(6+6 k^{3} z^{3}+k z\left(21+8 z+6 z^{2}+z^{3}\right)+k^{2} z^{2}\left(21+8 z+6 z^{2}+z^{3}\right)\right)} .
$$


We can specialize this formula to stars and paths. The star $\mathcal{S}_{n}$ having $n$ vertices can be written as the generalized star $G_{n-1,1}$, while the path $\mathcal{P}_{n}$ having $n$ vertices is the generalized star $G_{1, n-1}$. From this we find

$$
\beta^{*}\left(\mathcal{S}_{n}\right)=\frac{n^{3}+2 n^{2}-n-1}{n\left(n^{2}+3 n-3\right)}
$$

and

$$
\beta^{*}\left(\mathcal{P}_{n}\right)=\frac{4 n^{3}+6 n^{2}+4 n+1}{5 n\left(n^{2}+n+1\right)} .
$$

It is interesting to look at the extremal behaviour of $\beta^{*}\left(G_{k, z}\right)$ as we increase $k$ or $z$.

From (5) we see that, keeping $z \geq 1$ fixed,

$$
\lim _{k \rightarrow \infty} \beta^{*}\left(G_{k, z}\right)=1 .
$$

On the other side, keeping $k$ fixed we have

$$
\lim _{z \rightarrow \infty} \beta^{*}\left(G_{k, z}\right)=\frac{4}{5 k} .
$$

For stars and paths this becomes

$$
\lim _{n \rightarrow \infty} \beta^{*}\left(S_{n}\right)=1 \text { and } \lim _{n \rightarrow \infty} \beta^{*}\left(P_{n}\right)=\frac{4}{5} .
$$

This suggests that the parameter $\pi_{e}(M)$ provides a very good approximation to $\rho(M)$ for trees having a small maximal distance from the root compared to the number of vertices.

\section{Extremal properties of $\pi_{e}$ and majorization}

Let again $T$ be a rooted tree, with root $r$. Recall the notation $j \preceq i$, for vertices $i$ and $j$, which means that the path $P_{j}$ contains the path $P_{i}$, so $j$ is "below" or after $i$, seen from the root.

Let $\mathcal{T}_{n}$ denote the class of rooted trees with $n$ vertices. We identify two rooted trees $\left(T_{1}, r_{1}\right),\left(T_{2}, r_{2}\right)$ in $\mathcal{T}_{n}$ when there exists a rooted tree isomorphism from $T_{1}$ to $T_{2}$ (i.e. a graph isomorphism mapping $r_{1}$ to $r_{2}$ ). 
We are interested in the maximum and the minimum of $\pi_{e}$ in $\mathcal{T}_{n}$, so we define

$$
M_{n}\left(\pi_{e}\right)=\max _{T \in \mathcal{T}_{n}} \pi_{e}(T) \text {, and } m_{n}\left(\pi_{e}\right)=\min _{T \in \mathcal{T}_{n}} \pi_{e}(T) .
$$

Two special trees in $\mathcal{T}_{n}$ are the path $\mathcal{P}_{n}$ and the star $\mathcal{S}_{n}$. These turn out to be extreme in terms of the value of the parameter $\pi_{e}$, as the next result says.

Theorem 3.1. The maximum $M_{n}\left(\pi_{e}\right)$ is obtained for the path and the minimum $m_{n}\left(\pi_{e}\right)$ is obtained for the star, so

$$
\begin{aligned}
& M_{n}\left(\pi_{e}\right)=\pi_{e}\left(\mathcal{P}_{n}\right), \\
& m_{n}\left(\pi_{e}\right)=\pi_{e}\left(\mathcal{S}_{n}\right) .
\end{aligned}
$$

Moreover, if $T \in \mathcal{T}_{n}$ is different from $\mathcal{P}_{n}$ and $\mathcal{S}_{n}$, then $\pi_{e}(T)$ lies strictly between these extremal values.

Proof. Let, as usual, $P_{i}$ be the path between the root $r$ and vertex $i$ in $T$, where we may assume, by breadth-first-ordering from the root, that $\left|P_{i}\right| \leq i$ for each $i$. Then for all $i$ and $j$

$$
\left|P_{i} \cap P_{j}\right| \leq \min \left\{\left|P_{i}\right|,\left|P_{j}\right|\right\} .
$$

Therefore

$$
\begin{aligned}
\pi_{e}(T) & =\left(\frac{1}{n} \sum_{i}\left(\sum_{j}\left|P_{i} \cap P_{j}\right|\right)^{2}\right)^{1 / 2} \\
& \leq\left(\frac{1}{n} \sum_{i}\left(\sum_{j} \min \left\{\left|P_{i}\right|,\left|P_{j}\right|\right\}\right)^{2}\right)^{1 / 2} \\
& \leq\left(\frac{1}{n} \sum_{i}\left(\sum_{j} \min \{i, j\}\right)^{2}\right)^{\frac{1}{2}} \\
& =\pi_{e}\left(\mathcal{P}_{n}\right) .
\end{aligned}
$$

The last equality is because $\mathcal{P}_{n}$ satisfies (8) with equality, and $\left|P_{i}\right|=i$ for each $i$. In fact, $\mathcal{P}_{n}$ is the unique tree for which (8) holds with equality for all $i, j$.

Next, clearly, for the general tree $T$

$$
\left|P_{i} \cap P_{j}\right| \geq\left\{\begin{array}{l}
1 \text { for } i \text { and } j \text { distinct } \\
2 \text { for } i=j \neq r
\end{array}\right.
$$

and $\left|P_{r} \cap P_{r}\right|=1$. The only tree for which (9) holds with equality for all $i$ and $j$ is the star. 

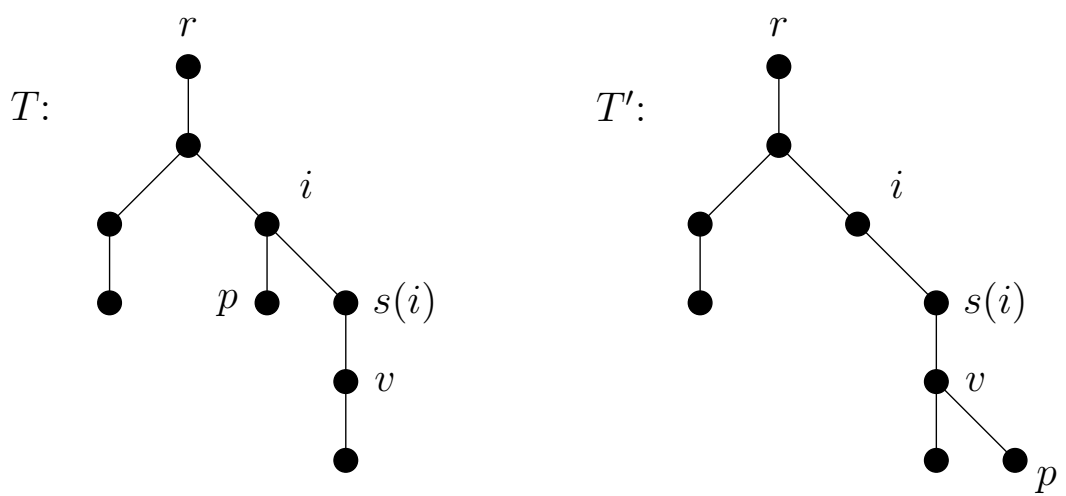

Figure 1: The trees $T$ and $T^{\prime}$; here $T(i, v)$ is the subtree induced by the vertices $i$ and $p$.

In Section 5 we compute the exact value of $\pi_{e}$ for these extremal trees.

Next, we investigate a certain operation on trees. Let $T \in \mathcal{T}_{n}$, and let $v \in V$. For each vertex $i \in P_{v}$ with $i \neq v$, let $T(i ; v)$ denote the subtree of $T$ induced by the vertex set $\{j: j \preceq i, j \npreceq s(i)\}$ where $s(i)$ denotes the first vertex after $i$ in the path $P_{v}$ seen from the root. Let $T^{\prime}$ be obtained by removing the subtree $T(i ; v)$ (except $i$ ) and attaching it at vertex $v$ (so the vertex $i$ of the subtree is identified with $v$ ). An example is shown in Figure 1 . Note that if the subtree $T(i ; v)$ only contains vertex $i$, then $T^{\prime}=T$. Otherwise, $T^{\prime} \neq T$, and we then say that $T^{\prime}$ is obtained from $T$ by moving a nontrivial subtree away from the root.

Lemma 3.2. Let $T \in \mathcal{T}_{n}$ and $T^{\prime}$ be obtained from $T$ by moving a nontrivial subtree away from the root. Then $\pi_{e}\left(T^{\prime}\right)>\pi_{e}(T)$.

Proof. The result follows by observing that, when we identify vertices in $T$ and $T^{\prime}$ in the natural way, then

$$
\left|P_{i}^{\prime} \cap P_{j}^{\prime}\right| \geq\left|P_{i} \cap P_{j}\right|
$$

for each $i$ and $j$, where $P_{i}$ and $P_{i}^{\prime}$ refers to $T$ and $T^{\prime}$, respectively. Here strict inequality holds, for instance, when $i$ and $j$ both belong to the subtree moved.

As an application of this lemma we will prove an interesting result for caterpillars. First, we introduce a version of the notion of majorization. A 
comprehensive treatment of majorization is given in the book [9], and majorization in connection with trees is investigated in [3]. Let $x=\left(x_{1}, x_{2}, \ldots, x_{t}\right)$ and $y=\left(y_{1}, y_{2}, \ldots, y_{t}\right)$ be real vectors. If

$$
\sum_{j=1}^{s} x_{j} \leq \sum_{j=1}^{s} y_{j} \quad(s \leq t)
$$

with equality for $s=t$, then we say that $x$ is majorized by $y$, and write $x \preceq^{*} y$. Note that this is different from classical majorization where similar inequalities hold for partial sums of the ordered components of the vectors.

Let $p_{1}, p_{2}, \ldots, p_{k}$ be nonnegative integers where $p_{k}=0$ and define the vector $p=\left(p_{1}, p_{2}, \ldots, p_{k}\right)$. We let $C(p)$ denote the rooted caterpillar consisting of a (central) path of length $k$ (i.e., $k$ vertices) and $p_{i}$ pendant vertices attached to vertex $i$ in this path $(i \leq k)$. The root is the first vertex in the central path (associated with $\left.p_{1}\right)^{1}$. Similarly, let $q_{1}, q_{2}, \ldots, q_{k}$ be nonnegative integers where $q_{k}=0$ and define the vector $q=\left(q_{1}, q_{2}, \ldots, q_{k}\right)$.

The following result shows how a majorization $p \preceq^{*} q$ for the vector $p$ affects the value of $\pi_{e}$.

Theorem 3.3. Let $p$ and $q$ be as above and assume $p \preceq^{*} q$. Then

$$
\pi_{e}(C(p)) \geq \pi_{e}(C(q))
$$

and strict inequality holds unless $p=q$.

In particular, for a nonnegative integer $m$ and any vector $p$ (as above) with $\sum_{j} p_{j}=m$, we have

$$
\pi_{e}(C((m, 0, \ldots, 0,0))) \leq \pi_{e}(C(p)) \leq \pi_{e}(C((0,0, \ldots, m, 0))) .
$$

Proof. If $p=q$, there is nothing to prove, so assume they are different. Let $i_{1}$ be smallest possible such that $\sum_{j=1}^{i_{1}} p_{j}<\sum_{j=1}^{i_{1}} q_{j}$. Therefore $p_{i}=q_{i}$ for $i<i_{1}$, and $p_{i_{1}}<q_{i_{1}}$. Next, let $i_{2}>i_{1}$ be smallest possible such that $p_{i_{2}}>q_{i_{2}}$. Such an $i_{2}$ must exist as $\sum_{j=1}^{k-1} p_{j}=\sum_{j=1}^{k-1} q_{j}$. Moreover, by these choices,

$$
\sum_{j=1}^{i} p_{j}<\sum_{j=1}^{i} q_{j} \quad\left(i=i_{1}, i_{1}+1, \ldots, i_{2}-1\right) .
$$

\footnotetext{
${ }^{1}$ If $p_{k} \geq 1$ we can define the new vector of nonnegative integers $p^{\prime}=$ $\left(p_{1}, p_{2}, \ldots, p_{k-1}, p_{k}-1,0\right)$. The rooted caterpillars $C(p)$ and $C\left(p^{\prime}\right)$ are clearly isomorphic as rooted trees. This is why we can assume, w.l.o.g., that $p_{k}=0$. We point out that this does not apply to $p_{1}, p_{2}, \ldots, p_{k-1}$.
} 
Define $q^{\prime}=\left(q_{1}^{\prime}, q_{2}^{\prime}, \ldots, q_{k}^{\prime}\right)$ from $q$ by letting $q_{i_{1}}^{\prime}=q_{i_{1}}-1, q_{i_{2}}^{\prime}=q_{i_{2}}+1$, and $q_{i}^{\prime}=q_{i}$ otherwise. Then, it is easy to check that

$$
p \preceq^{*} q^{\prime} \preceq^{*} q .
$$

By moving the subtree consisting of a leaf at vertex $i_{1}$ to vertex $i_{2}$, it follows from Lemma 3.2 that $\pi_{e}\left(C\left(q^{\prime}\right)\right)>\pi_{e}(C(q))$. We now replace $q$ by $q^{\prime}$ and repeat this procedure. After a finite number of such operations we obtain $q^{\prime}=p$, and the first result then follows from the corresponding ordering of the $\pi_{e}$ of these caterpillars.

The final statement follows from the first part of the proof as for any such $p$ the following majorizations hold

$$
(0, \ldots, 0, m, 0) \preceq^{*} p \preceq^{*}(m, 0, \ldots, 0) .
$$

\section{Upper bounds on the Perron value and $\rho_{c}$}

It is interesting to provide some bounds on $\rho$ and $\rho_{c}$, in order to see how distances and other basic parameters of the rooted tree affect these.

Let as usual $T$ be a rooted tree with $n$ vertices labeled as $1,2, \ldots, n$, where vertex 1 is the root, and let $M=\left[m_{i j}\right]$ be its bottleneck matrix. We get an upper bound on $\rho_{c}(M)$ by using the Lagrange identity (see [13]) on a suitable expression. Recall that, for a vertex $i$, the weight $\sigma_{i}$ is defined as $\sigma_{i}=\sum_{j: j \preceq i} d_{j}$. For the root this becomes $\sigma_{1}=\sum_{j=1}^{n} d_{j}=d \cdot e$, where $d$ is the distance vector of $T$ and $e$ is the all ones vector.

\section{Theorem 4.1.}

$$
\rho_{c}(M)=n \sum_{i} \frac{\sigma_{i}^{2}}{\sigma_{1}^{2}+\Delta} \leq n \sum_{i}\left(\frac{\sigma_{i}}{\sigma_{1}}\right)^{2}
$$

where $\Delta=(1 / 2) \sum_{i} \sum_{j}\left(d_{i}-d_{j}\right)^{2}$. In particular, $\rho_{c}(M) \leq n^{2}$.

Proof. Let $\sigma=\left(\sigma_{1}, \sigma_{2}, \ldots, \sigma_{n}\right)$ and $d=\left(d_{1}, d_{2}, \ldots, d_{n}\right)$. The Lagrange identity (see [13]) for vectors $x=\left(x_{1}, x_{2}, \ldots, x_{n}\right)$ and $y=\left(y_{1}, y_{2}, \ldots, y_{n}\right)$ says that $(x \cdot y)^{2}=\|x\|^{2}\|y\|^{2}-(1 / 2) \sum_{i} \sum_{j}\left(x_{i} y_{j}-x_{j} y_{i}\right)^{2}$; this is easy to 
verify by computing the double sum of squares. Using this with $x=d$ and $y=e$ gives

$$
\sigma_{1}^{2}=(d \cdot e)^{2}=\|d\|^{2} \cdot n-(1 / 2) \sum_{i} \sum_{j}\left(d_{i}-d_{j}\right)^{2}=n\|d\|^{2}-\Delta .
$$

Therefore

$$
\rho_{c}(M)=\frac{\|\sigma\|^{2}}{\|d\|^{2}}=\frac{n\|\sigma\|^{2}}{\sigma_{1}^{2}+\Delta}=n \sum_{i} \frac{\sigma_{i}^{2}}{\sigma_{1}^{2}+\Delta} \leq n \sum_{i}\left(\frac{\sigma_{i}}{\sigma_{1}}\right)^{2},
$$

and the result follows.

Numerical experiments suggest that the bound above is often quite good.

The next result gives an upper bound on the spectral radius $\rho(M)$, and therefore also on $\rho_{c}(M)$. It involves the sum norm, so recall that, for the distance vector $d,\|d\|_{1}=\sum_{i} d_{i}=\sigma_{1}$. Moreover, $\|d\|_{\infty}=\max _{i} d_{i}$ is the maximum distance from the root.

\section{Theorem 4.2.}

$$
\rho(M) \leq\|d\|_{1}+\|d\|_{\infty}-n
$$

Proof. Any consistent matrix norm gives an upper bound on the spectral radius of a matrix, and we therefore consider the infinity norm of the bottleneck matrix

$$
\rho(M) \leq\|M\|_{\infty}=\max _{i} \sum_{j} m_{i j}
$$

Let now $i$ be such that $\sum_{j} m_{i j}=\|M\|_{\infty}$. Consider a vertex $j$. If $j \notin P_{i}$, then

$$
m_{i j}=\left|P_{i} \cap P_{j}\right| \leq\left|P_{j}\right|-1=d_{j}-1 .
$$

On the other hand, if $j \in P_{i}$, then clearly $m_{i j}=d_{j}$. Hence,

$$
\begin{aligned}
\rho(M) & \leq\|M\|_{\infty} \\
& =\sum_{j \in P_{i}} m_{i j}+\sum_{j \notin P_{i}} m_{i j} \\
& \leq \sum_{j \in P_{i}} d_{j}+\sum_{j \notin P_{i}}\left(d_{j}-1\right) \\
& =\sum_{j} d_{j}-\left(n-\left|P_{i}\right|\right) \\
& \leq\|d\|_{1}-n+\|d\|_{\infty} .
\end{aligned}
$$


We notice that the bound in Theorem 4.2 is sharper than the bound $\rho(M) \leq\|d\|_{1}$, since the quantity $\|d\|_{\infty}-n$ is always nonpositive. In the special case of a star or a path the bound in Theorem 4.2 is very good, in fact it gives

$$
\rho\left(\mathcal{S}_{n}\right) \leq n+1, \text { and } \rho\left(\mathcal{P}_{n}\right) \leq \frac{n^{2}}{2}+\frac{n}{2}
$$

Recall that the exact Perron values for stars and paths are known (see [2]), and they are

$$
\begin{aligned}
& \rho\left(\mathcal{S}_{n}\right)=(1 / 2)\left(n+1+\sqrt{n^{2}+2 n-3}\right), \\
& \rho\left(\mathcal{P}_{n}\right)=\frac{1}{2}\left(1-\cos \left(\frac{\pi}{2 n+1}\right)\right)^{-1} .
\end{aligned}
$$

Finally, we apply Theorem 4.2 to rooted caterpillars and obtain the following result.

Corollary 4.3. Consider the rooted caterpillar $C(p)$ where $p=\left(p_{1}, p_{2}, \ldots, p_{k}\right)$ where $p_{k}=0$. Then

$$
\rho(C(p)) \leq\|d\|_{1}-\|p\|_{1} .
$$

Proof. This follows from Theorem 4.2 by noting that the distance vector of $C(p)$ satisfies $\|d\|_{\infty}-n=k-\left(k+\sum_{i=1}^{k-1} p_{i}\right)=-\sum_{i=1}^{k-1} p_{i}=-\|p\|_{1}$.

\section{Exact values for the combinatorial Perron parameters of certain trees}

In this section we compute the exact values of the combinatorial Perron parameters for certain classes of rooted trees. First we consider $\pi_{e}$ for the rooted star $\mathcal{S}_{n}$ and the rooted path $\mathcal{P}_{n}$, as defined at the end of the Introduction. Moreover, the exact value for $\rho_{c}(T)$ when $T$ is a broom tree is obtained. Additionally, exact values for $\pi_{d}\left(\mathcal{S}_{n}\right)$ and $\pi_{d}\left(P_{n}\right)$ are given.

Recall that $\mathcal{S}_{n}$ denotes the star with $n$ vertices having the central vertex as root; therefore, we label the central vertex as 1 and the remaining vertices as $2,3, \ldots, n$ in no specific order. Also, we denote by $\mathcal{P}_{n}$ the path with $n$ vertices having one of the endpoints as root; its vertices are labeled from 1 (the root) to $n$ (the other endpoint) in the natural way. For instance, the 
bottleneck matrix for the star $\mathcal{S}_{4}$ is

$$
M=\left[\begin{array}{llll}
1 & 1 & 1 & 1 \\
1 & 2 & 1 & 1 \\
1 & 1 & 2 & 1 \\
1 & 1 & 1 & 2
\end{array}\right]
$$

\section{Proposition 5.1.}

$$
\pi_{e}\left(\mathcal{S}_{n}\right)=\sqrt{\frac{n^{3}+2 n^{2}-n-1}{n}}
$$

and

$$
\pi_{e}\left(\mathcal{P}_{n}\right)=\sqrt{\frac{4 n^{5}+10 n^{4}+10 n^{3}+5 n^{2}+n}{30 n}} .
$$

Proof. For the star $\mathcal{S}_{n}$ we have $\left|P_{i} \cap P_{j}\right|=2$ when $i=j \neq 1$, and otherwise $\left|P_{i} \cap P_{j}\right|=1$. Thus, the expression follows. Next, consider the path $\mathcal{P}_{n}$. Here, we use the known formulas for $\sum_{i=1}^{n} i^{s}$ for $s=2,3,4$. Let

$$
\Psi=\sum_{i=1}^{n}\left((n-i) i+\sum_{j=1}^{i} j\right)^{2} .
$$

Then $\Psi=\Psi_{1}+2 \Psi_{2}+\Psi_{3}$, where

$$
\begin{aligned}
& \Psi_{1}=\sum_{i=1}^{n}\left(\sum_{j=1}^{i} j\right)^{2}=\frac{3 n^{5}+15 n^{4}+25 n^{3}+15 n^{2}+2 n}{60} \\
& \Psi_{2}=\sum_{i=1}^{n}\left[\left(\sum_{j=1}^{i} j\right)(n-i) i\right]=\frac{3 n^{5}+5 n^{4}-5 n^{3}-5 n^{2}+2 n}{120} \\
& \Psi_{3}=\sum_{i=1}^{n}(n-i)^{2} i^{2}=\frac{n^{5}-n}{30} .
\end{aligned}
$$

We have that $\pi_{e}\left(\mathcal{P}_{n}\right)=\left(\frac{1}{n} \Psi\right)^{1 / 2}$, which gives the desired expression.

We now consider the broom tree $B(x, y)$ which is obtained from the path $\mathcal{P}_{y}$ by adding $x$ vertices and attaching each of these to the root of the path; therefore, it has $x+y$ vertices. The root of $B(x, y)$ corresponds to the root of the path $\mathcal{P}_{y}$. 


\section{Proposition 5.2.}

$\rho_{c}(B(x, y))=\frac{4 y^{5}+10 y^{4}+10 y^{3}+(60 x+5) y^{2}+(60 x+1) y+120 x^{2}+120 x}{10 y^{3}+15 y^{2}+120 x+5 y}$.

Proof. Let the $y$ vertices of $B(x, y)$ corresponding to the path $\mathcal{P}_{y}$ be denoted by $p_{1}, p_{2}, \ldots, p_{y}$, so that $p_{1}$ is the root of $B(x, y)$ and $p_{y}$ is the farthest vertex from the root. Also, let the $x$ additional pendant vertices be denoted by $q_{1}, q_{2}, \ldots, q_{x}$. We find the following expressions for the distances and weights of vertices in $B(x, y)$ :

$$
\begin{array}{llll}
d_{p_{1}}=1, & \sigma_{p_{1}}=\sum_{j=1}^{y} j+2 x ; & & \\
d_{p_{i}}=i, & \sigma_{p_{i}}=\sum_{j=i}^{y} j, & & \text { for } i=2,3, \ldots, y ; \\
d_{q_{i}}=2, & \sigma_{q_{i}}=2, & & \text { for } i=1,2, \ldots, x .
\end{array}
$$

Therefore,

$$
\|d\|^{2}=4 x+\frac{2 y^{3}+3 y^{2}+y}{6}
$$

and

$$
\begin{aligned}
\|\sigma\|^{2} & =4 x+\left(\sum_{j=1}^{y} j+2 x\right)^{2}+\sum_{i=2}^{y}\left(\sum_{j=i}^{y} j\right)^{2} \\
& =4 x+2 x y^{2}+2 x y+4 x^{2}+\frac{1}{6} y^{2}+\frac{1}{30} y+\frac{2}{15} y^{5}+\frac{1}{3} y^{4}+\frac{1}{3} y^{3} .
\end{aligned}
$$

Plugging (12) and (13) into the equation

$$
\rho_{c}(B(x, y))=\frac{\|\sigma\|^{2}}{\|d\|^{2}}
$$

yields (11).

When $y=2$ the expression for $\rho_{c}(B(x, 2))$ is exactly the expression already obtained in [2] for the star and, when $x=0$, the expression for $\rho_{c}(B(0, y))$ is the one obtained in [2] for the path. Moreover, for fixed $y$, when $x$ is large compared to $y, \rho_{c}(B(x, y)) \approx x$. Similarly, for fixed $x$, when $y$ is large compared to $x, \rho_{c}(B(x, y)) \approx(2 / 5) y^{2}$.

Finally, we give the exact value for the parameter $\pi_{d}(T)$, when $T$ is the rooted star $\mathcal{S}_{n}$ or the rooted path $\mathcal{P}_{n}$. 


\section{Proposition 5.3.}

$$
\begin{aligned}
& \pi_{d}\left(\mathcal{S}_{n}\right)=\sqrt{\frac{4 n^{3}+4 n^{2}-7 n}{4 n-3}}, \\
& \pi_{d}\left(\mathcal{P}_{n}\right)=\sqrt{\frac{68 n^{4}+136 n^{3}+133 n^{2}+65 n+18}{420}} .
\end{aligned}
$$

Proof. Recall that, for a rooted tree $T$ having distance vector $d$ and bottleneck matrix $M=\left[m_{i j}\right], \pi_{d}(T)=\frac{\|M d\|}{\|d\|}$.

If $T$ is the star $\mathcal{S}_{n}$, then $d=(1,2, \ldots, 2)$, and

$$
m_{i j}= \begin{cases}2 & \text { if } i=j \neq 1 \\ 1 & \text { otherwise }\end{cases}
$$

Hence, $M d=(2 n-1,2 n+1, \ldots, 2 n+1)$. We obtain that $\|d\|=\sqrt{4 n-3}$ and $\|M d\|=\sqrt{4 n^{3}+4 n^{2}-7 n}$. The result then follows.

If $T$ is the path $\mathcal{P}_{n}$, then $d=(1,2, \ldots, n)$, and $m_{i j}=\min \{i, j\}$. Hence, the $i$ 'th entry of $M d$ can be written as

$$
(M d)_{i}=\sum_{j=1}^{n} m_{i j} d_{j}=\sum_{j=1}^{n} \min \{i, j\} \cdot j=\sum_{j \leq i} j^{2}+\sum_{j>i} i j=-\frac{1}{6} i\left(i^{2}-3 n^{2}-3 n-1\right) .
$$

We obtain that $\|d\|=\sqrt{\frac{2 n^{3}+3 n^{2}+n}{6}}$ and

$$
\begin{aligned}
\|M d\| & =\sqrt{\sum_{i=1}^{n}\left(-\frac{1}{6} i\left(i^{2}-3 n^{2}-3 n-1\right)\right)^{2}} \\
& =\sqrt{\frac{136 n^{7}+476 n^{6}+742 n^{5}+665 n^{4}+364 n^{3}+119 n^{2}+18 n}{2520}} .
\end{aligned}
$$

This yields

$$
\pi_{d}\left(\mathcal{P}_{n}\right)=\frac{\|M d\|}{\|d\|}=\sqrt{\frac{68 n^{4}+136 n^{3}+133 n^{2}+65 n+18}{420}} .
$$

We know the exact values of $\rho_{c}$ for the star and the path from [2]:

$$
\rho_{c}\left(\mathcal{S}_{n}\right)=n+\frac{3 n-3}{4 n-3}, \quad \rho_{c}\left(\mathcal{P}_{n}\right)=\frac{2 n^{2}+2 n+1}{5} .
$$

Asymptotically, for the path $\mathcal{P}_{n}$ when $n$ is large, one can verify that $\pi_{e}\left(\mathcal{P}_{n}\right)<\rho_{c}\left(\mathcal{P}_{n}\right)<\pi_{d}\left(\mathcal{P}_{n}\right)$. For the star $\mathcal{S}_{n}$, however, the values of the three parameters are asymptotically equal (to $n$ ). 


\section{An extension property for $\rho_{c}$}

The goal of this section is to prove an extension result for the combinatorial Perron value $\rho_{c}$, stating that this parameter increases when an edge is added at the root, and the root is "moved" to the new vertex. We point out that a similar property also holds for the Perron value $\rho$ (but that property is very easy to prove). The theorem we obtain will be used in the next section concerning centers.

Let $(T, r)$ be a rooted tree. We define its extension at the root to be the rooted tree $(\tilde{T}, \tilde{r})$ obtained from $T$ by adding a new vertex $\tilde{r}$ and an edge $\tilde{r} r$; the root of $\tilde{T}$ is $\tilde{r}$. Note that the distance of a vertex $v \neq \tilde{r}$ in $\tilde{T}$ is one larger than the corresponding distance in $T$.

The remaining part of this section is devoted to the proof of the following theorem, by establishing several intermediate results.

Theorem 6.1 (Extension property). Let $(T, r)$ be a rooted tree and let $(\tilde{T}, \tilde{r})$ be its extension at the root. Then

$$
\rho_{c}(\tilde{T})>\rho_{c}(T)
$$

The following lemma ([11]), which is straightforward to prove, turns out to be very useful in several proofs concerning $\rho_{c}(T)$.

Lemma 6.2. Let $a_{1}, a_{2}, b_{1}, b_{2}>0$ and suppose that $a_{1} / b_{1} \neq a_{2} / b_{2}$. Then

$$
\min \left\{\frac{a_{1}}{b_{1}}, \frac{a_{2}}{b_{2}}\right\}<\frac{a_{1}+a_{2}}{b_{1}+b_{2}}<\max \left\{\frac{a_{1}}{b_{1}}, \frac{a_{2}}{b_{2}}\right\} .
$$

Let $(T, r)$ be a rooted tree with vertex set $V$ and bottleneck matrix $M=$ $\left[m_{i j}\right]$, and let $n=|V|$. We define its descendant vector $\kappa=\left(\kappa_{1}, \kappa_{2}, \ldots, \kappa_{n}\right)$ as follows:

$$
\kappa_{i}=|\{j \in V: j \preceq i\}| \quad(i \in V) .
$$

Thus, $\kappa$ is the row sum vector of the path matrix $N$ of $T$. Let again $\sigma$ be the weight vector of $T$, so $\sigma_{i}=\sum_{j: j \preceq i} d_{j}$ for each $i \in V$. We define

$$
\beta_{T}:=\left(\sigma_{r}+n+1\right)^{2}+2 \kappa \cdot \sigma-\left(2 \sigma_{r}+n+1\right)\|M\|_{\infty}
$$

We point out that $\sigma_{r}$ denotes the weight of the root $r$ which, following our labeling convention, can also be referred to as $\sigma_{1}$. Note that, if the $g$-th row 
of the bottleneck matrix $M$ of $T$ attains the maximum row sum among all the rows of $M$, then $\sum_{v \in V} m_{g v}=\|M\|_{\infty}$. We want to show that $\beta_{T}>0$ for all trees, and this will lead to a proof of the extension property.

We first consider the broom tree (as defined in Section 5).

Proposition 6.3. Let $x \geq 0$ and $y \geq 1$ be integers, and consider the broom tree $B=B(x, y)$. Then $\beta_{B}>0$.

Proof. We use, for the vertices in $B$, the same labeling as in the proof of Proposition 5.2: $p_{1}, p_{2}, \ldots, p_{y}$ denote the vertices corresponding to the path $\mathcal{P}_{y}$, so that the root of $B$ is $r=p_{1}$. Also, $q_{1}, q_{2}, \ldots, q_{x}$ denote the $x$ additional pendant vertices.

The total number of vertices in $B$ is $n=x+y$. Let $g=p_{y}$ be the final vertex of the path $\mathcal{P}_{y}$ (the farthest from the root). If $x \geq 1$ and $y=1$, we have that $B(x, 1)=B(x-1,2)$. This means that we can assume without loss of generality that $g$ is a vertex attaining the maximum row sum among the rows of the bottleneck matrix $M$ of $B$. Let $\sigma$ and $\kappa$ denote the weight vector and the descendant vector of $B$ respectively. We have that

$$
\begin{array}{lll}
\sigma_{r}=\sum_{j=1}^{y} j+2 x, & \kappa_{r}=n ; & \\
\sigma_{p_{i}}=\sum_{j=i}^{y} j, & \kappa_{p_{i}}=y-i+1, & \text { for } i=2,3, \ldots, y ; \\
\sigma_{q_{i}}=2, & \kappa_{q_{i}}=1, & \text { for } i=1,2, \ldots, x .
\end{array}
$$

We compute

$$
\sigma_{r}=2 x+\sum_{j=1}^{y} j=2 x+\frac{y^{2}}{2}+\frac{y}{2}
$$

and

$$
\begin{aligned}
\kappa \cdot \sigma & =2 x+n \sigma_{r}+\sum_{i=2}^{y}\left[(y-i+1) \sum_{j=i}^{y} j\right] \\
& =2 x+(x+y)\left(2 x+\frac{y^{2}}{2}+\frac{y}{2}\right)+\frac{1}{2} \sum_{i=2}^{y}\left[(y-i+1)\left(y^{2}+y-i^{2}+i\right)\right] \\
& =2 x^{2}+\frac{x y^{2}}{2}+\frac{5}{2}+2 x+\frac{5}{24} y^{4}+\frac{5}{12} y^{3}+\frac{7}{24} y^{2}+\frac{y}{12} .
\end{aligned}
$$

Moreover, letting $V$ be the vertex set of $B$ and denoting as usual as $P_{v}$ the path joining a vertex $v$ to the root, we have that

$$
\begin{aligned}
\|M\|_{\infty} & =\sum_{v \in V} m_{g v}=\sum_{v \in V}\left|P_{g} \cap P_{v}\right|=\sum_{i=1}^{y}\left|P_{g} \cap P_{p_{i}}\right|+\sum_{i=1}^{x}\left|P_{g} \cap P_{q_{i}}\right| \\
& =\sum_{i=1}^{y} i+\sum_{i=1}^{x} 1=x+\frac{y^{2}}{2}+\frac{y}{2}
\end{aligned}
$$


Inserting these results into expression (15) yields

$$
\beta_{B}=1+9 x+8 x^{2}+\frac{8}{3} y+\frac{19}{2} x y+\frac{7}{3} y^{2}+\frac{x y^{2}}{2}+\frac{5}{6} y^{3}+\frac{y^{4}}{6}
$$

which is clearly strictly positive for each nonnegative value of $x$ and $y$.

We consider rooted caterpillars and, hereafter, use the simplified notation $C\left(n_{1}, \ldots, n_{p}\right)$, where $n_{p}=0$. We recall that the root $r$ of $C\left(n_{1}, \ldots, n_{p}\right)$ is the first vertex in its central path, associated with $n_{1}$.

Proposition 6.4. Let $C=C\left(n_{1}, \ldots, n_{p}\right)$ be a rooted caterpillar.

Then $\beta_{C}>0$.

Proof. Let $g$ be the final vertex of the central path of $C$ (the farthest from the root). We notice that $g$ is a vertex attaining the maximum row sum among the rows of the bottleneck matrix $M$ of $C$. We prove the result by using a certain tree modification procedure. Suppose that $C$ is not a broom. Then the integer

$$
s:=\max \left\{i: 1<i<p, n_{i}>0\right\}
$$

is well-defined. Define the new rooted caterpillar $\tilde{C}=C\left(n_{1}, \ldots, n_{s-2}, n_{s-1}+\right.$ $\left.1, n_{s}-1, n_{s+1}, \ldots, n_{p}\right)$. In other words, $\tilde{C}$ is the caterpillar obtained from $C$ by taking a leaf $c$ at distance $s+1$, deleting the unique edge $b c$ incident to $c$ and adding the edge $a c$ where $a$ is the father (ascendant) of $b$. We claim that

$$
\beta_{\tilde{C}}<\beta_{C} .
$$

Let us denote respectively by $\tilde{\sigma}, \tilde{\kappa}$ and $\tilde{M}$ the weight vector, the descendant vector and the bottleneck matrix of $\tilde{C}$, and set $x:=s+1$.

We have

$$
\begin{aligned}
& \tilde{\kappa}_{v}= \begin{cases}\kappa_{v} & \text { if } v \neq b, \\
\kappa_{v}-1 & \text { if } v=b,\end{cases} \\
& \tilde{\sigma}_{v}= \begin{cases}\sigma_{v} & \text { if } c \npreceq v, \\
\sigma_{v}-1 & \text { if } c \preceq v, v \neq b, \\
\sigma_{v}-x & \text { if } v=b .\end{cases}
\end{aligned}
$$


Therefore

$$
\begin{aligned}
\tilde{\kappa} \cdot \tilde{\sigma} & =\sum_{c \npreceq v} \kappa_{v} \sigma_{v}+\sum_{\substack{c \preceq v \\
v \neq b}} \kappa_{v}\left(\sigma_{v}-1\right)+\left(\kappa_{b}-1\right)\left(\sigma_{b}-x\right) \\
& =\kappa \cdot \sigma-\sum_{\substack{c \preceq v \\
v \neq b}} \kappa_{v}-\sigma_{b}-x \kappa_{b}+x .
\end{aligned}
$$

Looking at the bottleneck matrices $M=\left[m_{i j}\right]$ and $\tilde{M}=\left[\tilde{m}_{i j}\right]$, we see that $g$ is a vertex attaining the maximum row sum among the rows of $\tilde{M}$, too. Let $V=V(C)$. Set

$$
m:=\sum_{v \in V} m_{g v}=\|M\|_{\infty} \quad \text { and } \quad \tilde{m}:=\sum_{v \in V} \tilde{m}_{g v}=\|\tilde{M}\|_{\infty} .
$$

We have that $\tilde{m}=m-1$. Therefore

$$
\begin{aligned}
\beta_{\tilde{C}}= & \left(\tilde{\sigma}_{r}+n+1\right)^{2}+2 \tilde{\kappa} \cdot \tilde{\sigma}-\left(2 \tilde{\sigma}_{r}+n+1\right) \tilde{m} \\
= & \left(\left(\sigma_{r}+n+1\right)-1\right)^{2}+2\left(\kappa \cdot \sigma-\sum_{\substack{c \preceq v \\
v \neq b}} \kappa_{v}-\sigma_{b}-x \kappa_{b}+x\right) \\
& -\left(\left(2 \sigma_{r}+n+1\right)-2\right)(m-1) \\
= & \beta_{C}+1-2 \sigma_{r}-2 n-2-2 \sum_{\substack{c \preceq v \\
v \neq b}} \kappa_{v}-2 \sigma_{b}-2 x \kappa_{b}+2 x \\
& +2 \sigma_{r}+n+1+2 m-2 \\
= & \beta_{C}-2 \sum_{\substack{c \preceq v \\
v \neq b}} \kappa_{v}-2 \sigma_{b}-2 x \kappa_{b}+2 m-n+2 x-2 .
\end{aligned}
$$

The goal now is to show that

$$
A:=-2 \sum_{\substack{c \preceq v \\ v \neq b}} \kappa_{v}-2 \sigma_{b}-2 x \kappa_{b}+2 m-n+2 x-2<0 .
$$

This suffices to conclude that claim (17) is true. In order to achieve this we convert the quantities in (18) into expressions involving the numbers 
$n_{1}, n_{2}, \ldots, n_{p}$. First we see that the expression of $m$ is particularly simple, thanks to the caterpillar-structure of $C$ :

$$
m=\sum_{i=1}^{p} i\left(n_{i}+1\right) .
$$

Next, we notice that, if $v$ is a vertex belonging to the central path of $C$, then its number of descendants is:

$$
\kappa_{v}=\sum_{i=d_{v}}^{p}\left(n_{i}+1\right) .
$$

Therefore, using that $d_{a}=x-2$,

$$
\begin{aligned}
\sum_{\substack{c \preceq v \\
v \neq b}} \kappa_{v} & =\kappa_{c}+\sum_{a \preceq v} \kappa_{v} \\
& =1+\sum_{a \preceq v} \kappa_{v} \\
& =1+\sum_{j=1}^{x-2} \sum_{i=j}^{p}\left(n_{i}+1\right) \\
& =1+\sum_{j=1}^{p} \sum_{i=j}^{p}\left(n_{i}+1\right)-\sum_{j=x-1}^{p} \sum_{i=j}^{p}\left(n_{i}+1\right) \\
& =1+\sum_{i=1}^{p} i\left(n_{i}+1\right)-\sum_{i=x-1}^{p}(i-x+2)\left(n_{i}+1\right) \\
& =1+m-\sum_{i=x-1}^{p}(i-x+2)\left(n_{i}+1\right) .
\end{aligned}
$$


By (16), we know that $n_{x}=n_{x+1}=\cdots=n_{p}=0$. Therefore,

$$
\begin{aligned}
\sum_{\substack{c \preceq v \\
v \neq b}} \kappa_{v} & =1+m-(x-1-x+2)\left(n_{x-1}+1\right)-\sum_{i=x}^{p}(i-x+2) \\
& =m-n_{x-1}-\sum_{i=x}^{p}(i-x+2) \\
& =m-n_{x-1}-\frac{p^{2}+p-x^{2}+x}{2}+p x-x^{2}+x-2 p+2 x-2 \\
& =m-n_{x-1}-\frac{p^{2}}{2}-\frac{5}{2} p-\frac{x^{2}}{2}+\frac{5}{2} x+p x-2 .
\end{aligned}
$$

Finally,

$$
\begin{aligned}
\sigma_{b} & =x n_{x-1}+\sum_{i=x-1}^{p} i \\
& =x n_{x-1}+\frac{p^{2}+p-x^{2}+3 x-2}{2}
\end{aligned}
$$

and

$$
\begin{aligned}
\kappa_{b} & =n_{x-1}+p-(x-1)+1 \\
& =n_{x-1}+p-x+2 .
\end{aligned}
$$

Inserting all these expressions into (18) gives

$$
\begin{aligned}
A= & -2\left(m-n_{x-1}-\frac{p^{2}}{2}-\frac{5}{2} p-\frac{x^{2}}{2}+\frac{5}{2} x+p x-2\right)-2\left(x n_{x-1}+\frac{p^{2}+p-x^{2}+3 x-2}{2}\right) \\
& -2 x\left(n_{x-1}+p-x+2\right)+2 m-n+2 x-2 \\
= & 2 n_{x-1}+4 p+4 x^{2}-10 x-4 p x+4-4 x n_{x-1}-n \\
< & 4 p+4 x^{2}-10 x-4 p x+4 .
\end{aligned}
$$

Let us study the polynomial function

$$
f(P, X):=4 P+4 X^{2}-10 X-4 P X+4
$$

in the unbounded region

$$
\mathcal{R}:=\left\{(P, X) \in \mathbb{R}^{2} \mid X \geq 1, P \geq X\right\} .
$$


We have that

$$
\frac{\partial f}{\partial P}(\hat{P}, \hat{X})=4-4 \hat{X} \leq 0 \quad((\hat{P}, \hat{X}) \in \mathcal{R})
$$

and

$$
f(\hat{X}, \hat{X})=-6 \hat{X}+4<0 \quad((\hat{X}, \hat{X}) \in \mathcal{R})
$$

This shows that $f$ is strictly negative in $\mathcal{R}$. Returning to $p$ and $x$ above, since $(p, x) \in \mathcal{R}$, we obtain

$$
f(p, x)=4 p+4 x^{2}-10 x-4 p x+4<0 .
$$

Combining (19) and (20) we obtain (18) and, consequently, (17).

We can now repeat this process of moving an edge upwards until, after a finite number of steps, we end up with a broom $B$, so that

$$
\beta_{C} \geq \beta_{B}
$$

Therefore, by applying Proposition 6.3, we complete the proof.

Proposition 6.5. Let $(T, r)$ be a rooted tree of $n$ vertices. Then $\beta_{T}>0$.

Proof. Let $V$ be the vertex set of $T$, let $M=\left[m_{i j}\right]$ be the bottleneck matrix of $T$, and choose a vertex $g$ of $T$ so that the corresponding row in $M$ maximizes the row sum. We claim that $g$ is a leaf. In fact, if $c$ is a child of $g$, then

$$
\sum_{v \in V} m_{c v}>\sum_{v \in V} m_{g v}
$$

thus contradicting the definition of $g$. Let now $P_{g}$ be the path connecting $g$ to the root $r$. If $T$ is not a caterpillar with central path $P_{g}$, we can find a leaf $a$ in $T$ such that its father $u$ does not lie in $P_{g}$. Let $v$ be the father of $u$. Consider the tree $\tilde{T}$ obtained from $T$ by replacing the edge $u a$ with the edge $v a$. Let us denote respectively by $\tilde{\sigma}, \tilde{\kappa}$ and $\tilde{M}$ the weight vector, the descendant vector and the bottleneck matrix of $\tilde{T}$. Note that the vertex set of $\tilde{T}$ is $V$.

We notice that $M \geq \tilde{M}$ (entrywise order). Moreover, the choice of $a$ assures that

$$
m_{g v}=\tilde{m}_{g v} \quad(v \in V) .
$$


This shows that $g$ is a vertex attaining the maximum row sum among the rows of $\tilde{M}$, too, and that

$$
\|\tilde{M}\|_{\infty}=\sum_{v \in V} \tilde{m}_{g v}=\sum_{v \in V} m_{g v}=\|M\|_{\infty}
$$

Let us denote this common value by $m$. We claim that

$$
\beta_{\tilde{T}}<\beta_{T}
$$

First, note that for every vertex $v \in V$ it holds that $\tilde{\kappa}_{v} \leq \kappa_{v}$ and $\tilde{\sigma}_{v} \leq \sigma_{v}$; hence,

$$
\tilde{\kappa} \cdot \tilde{\sigma} \leq \kappa \cdot \sigma
$$

We then observe that

$$
\begin{aligned}
\beta_{\tilde{T}} & =\left(\tilde{\sigma}_{r}+n+1\right)^{2}+2 \tilde{\kappa} \cdot \tilde{\sigma}-\left(2 \tilde{\sigma}_{r}+n+1\right)\|\tilde{M}\|_{\infty} \\
& =\left(\left(\sigma_{r}+n+1\right)-1\right)^{2}+2 \tilde{\kappa} \cdot \tilde{\sigma}-\left(\left(2 \sigma_{r}+n+1\right)-2\right) m \\
& =\left(\sigma_{r}+n+1\right)^{2}+1-2 \sigma_{r}-2 n-2+2 \tilde{\kappa} \cdot \tilde{\sigma}-\left(2 \sigma_{r}+n+1\right) m+2 m \\
& \leq\left(\sigma_{r}+n+1\right)^{2}+1-2 \sigma_{r}-2 n-2+2 \kappa \cdot \sigma-\left(2 \sigma_{r}+n+1\right) m+2 m \\
& =\beta_{T}-2 \sigma_{r}-2 n-1+2 m \\
& <\beta_{T}-2\left(\sigma_{r}-m\right) \\
& \leq \beta_{T}
\end{aligned}
$$

where the last inequality follows by observing that

$$
m_{i j} \leq d_{j} \quad(i, j \in V)
$$

and hence

$$
m=\sum_{j \in V} m_{g j} \leq \sum_{j \in V} d_{j}=\sigma_{r}
$$

This shows (21). We can iteratively apply this process until we end up with a caterpillar $C$ with central path $P_{g}$. We know, then, that

$$
\beta_{T} \geq \beta_{C}
$$

Applying Proposition 6.4 we conclude the proof. 
We now have the results needed to prove the main theorem of this section.

Proof of Theorem 6.1: Suppose $T$ has $n$ vertices, which we label with integers from 1 to $n$ (where 1 is the root). Then $\tilde{T}$ has $n+1$ vertices; we label the root $\tilde{r}$ of $\tilde{T}$ with 0 , and we label the other vertices of $\tilde{T}$ accordingly to the labels of corresponding vertices in $T$. Denote respectively as $d_{i}$ and $\sigma_{i}$ the distance and the weight of vertex $i$ in $T, i=1,2, \ldots, n$. Analogously, denote respectively as $\tilde{d}_{i}$ and $\tilde{\sigma}_{i}$ the distance and the weight of vertex $i$ in $\tilde{T}$, $i=0,1, \ldots, n$. Define also $d_{0}:=0, \sigma_{0}:=0$. Let

$$
\begin{aligned}
D & :=\sum_{i=0}^{n} d_{i}^{2}, & \tilde{D} & =\sum_{i=0}^{n} \tilde{d}_{i}^{2}, \\
S & :=\sum_{i=0}^{n} \sigma_{i}^{2}, & \tilde{S} & =\sum_{i=0}^{n} \tilde{\sigma}_{i}^{2} .
\end{aligned}
$$

Finally, let $\sigma=\left(\sigma_{1}, \sigma_{2}, \ldots, \sigma_{n}\right)$ and $\kappa=\left(\kappa_{1}, \kappa_{2}, \ldots, \kappa_{n}\right)$ be the weight vector and the descendant vector of $T$ respectively. Looking at the definition of the extension tree $\tilde{T}$, we note that

$$
\begin{array}{ll}
\tilde{d}_{i}=d_{i}+1 & \forall i=0, \ldots, n ; \\
\tilde{\sigma}_{i}=\sum_{j \preceq i} \tilde{d}_{j}=\sum_{j \preceq i}\left(d_{j}+1\right)=\sigma_{i}+\kappa_{i} & \forall i=1, \ldots, n ; \\
\tilde{\sigma}_{0}=\sum_{j=0}^{n} \tilde{d}_{j}=\sum_{j=0}^{n}\left(d_{j}+1\right)=\sigma_{1}+n+1 . &
\end{array}
$$

We get

$$
\begin{aligned}
\tilde{D}-D & =\sum_{i=0}^{n}\left(\tilde{d}_{i}^{2}-d_{i}^{2}\right)=\sum_{i=0}^{n}\left(\tilde{d}_{i}-d_{i}\right)\left(\tilde{d}_{i}+d_{i}\right) \\
& =\sum_{i=0}^{n}\left(2 d_{i}+1\right)=2 \sigma_{1}+n+1 ; \\
\tilde{S}-S & =\sum_{i=0}^{n}\left(\tilde{\sigma}_{i}^{2}-\sigma_{i}^{2}\right)=\sum_{i=0}^{n}\left(\tilde{\sigma}_{i}-\sigma_{i}\right)\left(\tilde{\sigma}_{i}+\sigma_{i}\right) \\
& =\tilde{\sigma}_{0}^{2}+\sum_{i=1}^{n}\left(\tilde{\sigma}_{i}-\sigma_{i}\right)\left(\tilde{\sigma}_{i}+\sigma_{i}\right)=\left(\sigma_{1}+n+1\right)^{2}+\sum_{i=1}^{n} \kappa_{i}\left(2 \sigma_{i}+\kappa_{i}\right) \\
& =\left(\sigma_{1}+n+1\right)^{2}+2 \kappa \cdot \sigma+\kappa \cdot \kappa .
\end{aligned}
$$


We then write

$$
\rho_{c}(\tilde{T})=\frac{\tilde{S}}{\tilde{D}}=\frac{S+(\tilde{S}-S)}{D+(\tilde{D}-D)}=\frac{S+\left[\left(\sigma_{1}+n+1\right)^{2}+2 \kappa \cdot \sigma+\kappa \cdot \kappa\right]}{D+\left[2 \sigma_{1}+n+1\right]} .
$$

Using Lemma 6.2 we see that

$$
\rho_{c}(\tilde{T})>\rho_{c}(T) \quad \Leftrightarrow \quad \frac{\left(\sigma_{1}+n+1\right)^{2}+2 \kappa \cdot \sigma+\kappa \cdot \kappa}{2 \sigma_{1}+n+1}>\rho_{c}(T) .
$$

Let us define

$$
\alpha:=\frac{\left(\sigma_{1}+n+1\right)^{2}+2 \kappa \cdot \sigma}{2 \sigma_{1}+n+1} .
$$

It suffices to prove that

$$
\alpha>\rho_{c}(T)
$$

because then the theorem follows.

Consider the quantity $\beta_{T}$ defined in (15). We know from Proposition 6.5 that it is strictly positive:

$$
\beta_{T}=\left(\sigma_{1}+n+1\right)^{2}+2 \kappa \cdot \sigma-\left(2 \sigma_{1}+n+1\right)\|M\|_{\infty}>0
$$

so

$$
\|M\|_{\infty}<\frac{\left(\sigma_{1}+n+1\right)^{2}+2 \kappa \cdot \sigma}{2 \sigma_{1}+n+1}=\alpha .
$$

Noting that $\rho_{c}(T) \leq \rho(T) \leq\|M\|_{\infty}<\alpha$, we conclude that (23) holds, and the proof is complete.

\section{Caterpillars and a new center}

In this section we study the combinatorial Perron value $\rho_{c}$ for (rooted) caterpillars, and establish some interesting properties that we use to introduce a new center concept for (unrooted) caterpillars.

By an unrooted caterpillar we mean a caterpillar $C$ where we ignore the root. Consider an unrooted caterpillar with $n \geq 2$ vertices. Let $P:=$ $v_{1} v_{2} \ldots v_{k}$ be a longest path in $C$. There may be several such paths, but this ambiguity plays no role in the following. So $k \geq 2$. For an ordered pair $(i, j)$ of adjacent vertices $i$ and $j$ in $P$, let $C^{i \rightarrow j}$ denote the rooted connected 
component of $C \backslash\{i\}$ that contains $j$, and has $j$ as root. Note that $C^{i \rightarrow j}$ is a rooted caterpillar. Define

$$
\rho_{c}^{i \rightarrow j}:=\rho_{c}\left(C^{i \rightarrow j}\right) .
$$

To give some intuition, we think of $\rho_{c}^{i \rightarrow j}$ as a "weight" of the subtree $C^{i \rightarrow j}$. So, for every edge $i j$ in $P$ we have the two subtrees $C^{i \rightarrow j}$ and $C^{j \rightarrow i}$, each with a weight (in this sense). It is natural, then, to look for some kind of "gravity center" of $C$, which should divide the caterpillar into two parts whose weights are almost equal. We now explore this idea more rigorously. For each edge $e=i j \in P$, define

$$
\Delta_{e}:=\left|\rho_{c}^{i \rightarrow j}-\rho_{c}^{j \rightarrow i}\right| .
$$

We set

$$
\Delta^{*}:=\min _{e \in P} \Delta_{e}
$$

and define

$$
E^{*}=\left\{e \in P: \Delta_{e}=\Delta^{*}\right\} .
$$

We shall prove the following result.

Theorem 7.1. Let $C$ be an unrooted caterpillar with more than one vertex. Then $E^{*}$ contains either a single edge ij, or two edges incident to a vertex $i$.

Based on this, we define the gravity center of $C$ to be $\{i, j\}$ (resp. $\{i\}$ ) in the two cases described in the theorem. To prove Theorem 7.1 we need two results, which we combine in Proposition 7.3. The first is the extension property stated in Theorem 6.1. The second is given in Proposition 7.2.

Proposition 7.2. Let $(T, r)$ be a rooted tree, and let $(\tilde{T}, r)$ be the rooted tree obtained by adding an edge at the root $r$, i.e., adding a vertex $u$ and an edge $r u$, and taking $r$ as the root of $\tilde{T}$. Then $\rho_{c}(\tilde{T})>\rho_{c}(T)$.

Proof. Given a vertex $v \in V(T)$, let $d_{v}$ and $\sigma_{v}$ denote its distance and its weight in $T$ respectively. Given a vertex $v \in V(\tilde{T})$, let $\tilde{d}_{v}$ and $\tilde{\sigma}_{v}$ denote its distance and its weight in $\tilde{T}$, respectively. Let

$$
\begin{aligned}
D & =\sum_{v \in V(T)} d_{v}^{2}, & \tilde{D} & =\sum_{v \in V(\tilde{T})} \tilde{d}_{v}^{2}, \\
S & =\sum_{v \in V(T)} \sigma_{v}^{2}, & \tilde{S} & =\sum_{v \in V(\tilde{T})} \tilde{\sigma}_{v}^{2} .
\end{aligned}
$$


Here $\tilde{T}$ is obtained from $T$ by adding a vertex $u$ and joining it to $r$. We take the root of $\tilde{T}$ to be $r$. Then, $\tilde{d}_{u}=2$, since there are two vertices, namely $r$ and $u$, in the path joining $u$ to $r$ in $\tilde{T}$. Also, $u$ is the unique vertex which belongs to $\tilde{T}$ but not to $T$. Moreover, the distance from the root $r$ of a vertex $v \neq u$ is the same in $T$ and in $\tilde{T}$. Then,

$\tilde{D}=\sum_{v \in V(\tilde{T})} \tilde{d}_{v}^{2}=\tilde{d}_{u}^{2}+\sum_{v \in V(\tilde{T}), v \neq u} \tilde{d}_{v}^{2}=\tilde{d}_{u}^{2}+\sum_{v \in V(T)} \tilde{d}_{v}^{2}=\tilde{d}_{u}^{2}+\sum_{v \in V(T)} d_{v}^{2}=\tilde{d}_{u}^{2}+D$

Regarding $\tilde{S}$, the weight of $r$ in $\tilde{T}$ is different from the weight of $r$ in $T$, since in the former case we have to take into account that we added a new vertex, $u$ :

$$
\tilde{\sigma}_{r}=\sum_{v \in V(\tilde{T})} \tilde{d}_{v}=\tilde{d}_{u}+\sum_{v \in V(\tilde{T}), v \neq u} \tilde{d}_{v}=2+\sum_{v \in V(T)} d_{v}=2+\sigma_{r}
$$

The weight in $\tilde{T}$ of each vertex $v \neq r, u$ is the same as its weight in $T$, because it is not affected by the fact that we have added $u$; this is because $u$ is not below $v$ in $\tilde{T}$, and therefore it is not counted when we compute $\tilde{\sigma}_{v}$. Notice also that $\tilde{\sigma}_{u}=\tilde{d}_{u}=2$, since the only vertex in $\tilde{T}$ which is below $u$ is $u$ itself. Therefore, we obtain

$$
\begin{aligned}
\tilde{S} & =\sum_{v \in V(\tilde{T})} \tilde{\sigma}_{v}^{2}=\tilde{\sigma}_{u}^{2}+\tilde{\sigma}_{r}^{2}+\sum_{v \in V(\tilde{T}), v \neq u, r} \tilde{\sigma}_{v}^{2}=\tilde{\sigma}_{u}^{2}+\tilde{\sigma}_{r}^{2}+\sum_{v \in V(T), v \neq r} \sigma_{v}^{2} \\
& =\tilde{\sigma}_{u}^{2}+\tilde{\sigma}_{r}^{2}+\left(S-\sigma_{r}^{2}\right)=2^{2}+\left(2+\sigma_{r}\right)^{2}+S-\sigma_{r}^{2} .
\end{aligned}
$$

This discussion gives

$\rho_{c}(\tilde{T})=\frac{\tilde{S}}{\tilde{D}}=\frac{\left(S-\sigma_{r}^{2}+\tilde{\sigma}_{r}^{2}\right)+\tilde{\sigma}_{u}^{2}}{D+\tilde{d}_{u}^{2}}=\frac{S-\sigma_{r}^{2}+\left(\sigma_{r}+2\right)^{2}+4}{D+4}=\frac{S+4 \sigma_{r}+8}{D+4}$.

We know from [2] that $\rho_{c}(T) \leq \sum_{v \in V(T)} d_{v}=\sigma_{r}$. Therefore

$$
\frac{4 \sigma_{r}+8}{4}=\sigma_{r}+2>\rho_{c}(T)=\frac{S}{D} .
$$

Applying Lemma 6.2,

$$
\rho_{c}(\tilde{T})=\frac{S+4 \sigma_{r}+8}{D+4}>\min \left\{\frac{S}{D}, \frac{4 \sigma_{r}+8}{4}\right\}=\frac{S}{D}=\rho_{c}(T) .
$$


Given two rooted caterpillars $C_{1}=C\left(n_{1}, \ldots, n_{k}\right)$ and $C_{2}=C\left(m_{1}, \ldots, m_{p}\right)$, we say that $C_{1}$ extends $C_{2}$ provided that

$$
\begin{aligned}
& k>p, \\
& n_{k-i}=m_{p-i} \quad(0 \leq i \leq p-1) .
\end{aligned}
$$

Proposition 7.3. Let $C_{1}$ and $C_{2}$ be rooted caterpillars such that $C_{1}$ extends $C_{2}$. Then

$$
\rho_{c}\left(C_{1}\right)>\rho_{c}\left(C_{2}\right) .
$$

Proof. Let $\mathcal{C}$ be the set of rooted caterpillars. Define the maps

$$
\begin{aligned}
\alpha: \mathcal{C} & \rightarrow \mathcal{C} \\
C\left(n_{1}, n_{2}, \ldots, n_{k}\right) & \mapsto C\left(n_{1}+1, n_{2}, \ldots, n_{k}\right)
\end{aligned}
$$

and

$$
\begin{aligned}
\beta: \mathcal{C} & \rightarrow \mathcal{C} \\
C\left(n_{1}, n_{2}, \ldots, n_{k}\right) & \mapsto C\left(0, n_{1}, n_{2}, \ldots, n_{k}\right) .
\end{aligned}
$$

Given $C \in \mathcal{C}$, Proposition 7.2 shows that

$$
\rho_{c}(\alpha(C))>\rho_{c}(C),
$$

while the extension property Theorem 6.1 shows that

$$
\rho_{c}(\beta(C))>\rho_{c}(C) .
$$

The proposition follows by observing that $C_{1}$ can be obtained from $C_{2}$ by applying $\alpha$ and $\beta$ to $C_{2}$ for a finite number of times; in other words, there exist $p \geq 1, \gamma_{1}, \ldots, \gamma_{p} \in\{\alpha, \beta\}$, such that

$$
C_{1}=\gamma_{1} \circ \cdots \circ \gamma_{p}\left(C_{2}\right) .
$$

Proof of Theorem 7.1: Suppose, for the sake of contradiction, that there exist two non-consecutive edges $i j$ and $x y$ in a longest path $P$ in $C$ 
both belonging to $E^{*}$. Suppose also that we are labeling vertices in $P$ so that $j$ lies between $i$ and $x$, and $x$ lies between $j$ and $y$. Let $w$ be the vertex in $P$ adjacent to $j$ other than $i$ (possibly $w=x$ ). Using Proposition 7.3 we see that

$$
\rho_{c}^{i \rightarrow j}>\rho_{c}^{j \rightarrow w}>\rho_{c}^{x \rightarrow y}
$$

and

$$
\rho_{c}^{y \rightarrow x}>\rho_{c}^{w \rightarrow j}>\rho_{c}^{j \rightarrow i}
$$

But then

$$
\rho_{c}^{i \rightarrow j}-\rho_{c}^{j \rightarrow i}>\rho_{c}^{j \rightarrow w}-\rho_{c}^{w \rightarrow j}>\rho_{c}^{x \rightarrow y}-\rho_{c}^{y \rightarrow x} .
$$

If $\rho_{c}^{j \rightarrow w}-\rho_{c}^{w \rightarrow j} \geq 0$, then (24) implies that

$$
\Delta_{j w}=\rho_{c}^{j \rightarrow w}-\rho_{c}^{w \rightarrow j}<\rho_{c}^{i \rightarrow j}-\rho_{c}^{j \rightarrow i}=\Delta_{i j},
$$

which contradicts the minimality of $\Delta_{i j}$; if $\rho_{c}^{j \rightarrow w}-\rho_{c}^{w \rightarrow j}<0$, then (24) implies that

$$
\Delta_{j w}=\rho_{c}^{w \rightarrow j}-\rho_{c}^{j \rightarrow w}<\rho_{c}^{y \rightarrow x}-\rho_{c}^{x \rightarrow y}=\Delta_{x y},
$$

which contradicts the minimality of $\Delta_{x y}$.

We conclude the section presenting a short computational analysis of our results on caterpillars.

Having two distinct notions for the center of an unrooted caterpillar $C$ its characteristic set and its gravity center - we implemented two algorithms to compute them. The first compares the Perron value $\rho$ of the bottleneck matrices associated to rooted subtrees of $C$; the second considers the parameter $\rho_{c}$ instead. Given that in the second case we do not have to deal with eigenvalues, we expect the algorithm computing the gravity center to be faster.

We tested the two algorithms - written in MATLAB - on randomly generated unrooted caterpillars having different numbers of vertices, and we report the results in Figure 2(a). It clearly emerges that the second algorithm is the most computationally efficient.

Besides the computational time, it is interesting to find out how close the characteristic set and the gravity center of an unrooted caterpillar are. Given that both these sets can consist of either one or two vertices, to define their distance $\delta$ we need to distinguish among 3 cases: 


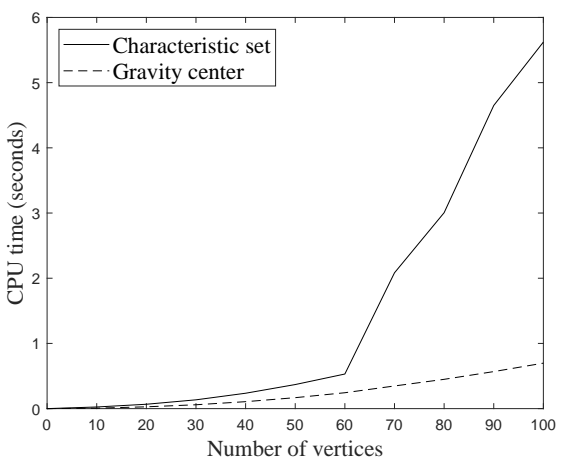

(a) Average CPU time

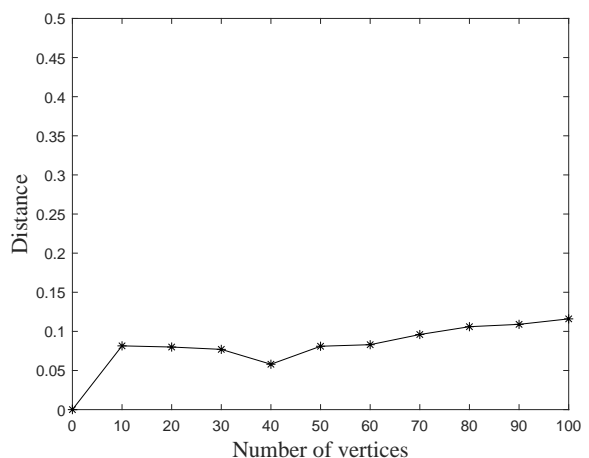

(b) Average distance

Figure 2: (a) shows the CPU time to find the characteristic set (in red) and the gravity center (in blue) of unrooted caterpillars. (b) shows the distance between the two centers. For a given number $n$ of vertices, the CPU time and the distance are both obtained as an average of 1000 randomly generated unrooted caterpillars with $n$ vertices.

- If both the centers consist of one vertex, then $\delta$ equals the number of edges in the path connecting them ( $\delta=0$ if the centers coincide).

- If both the centers consist of two vertices, then $\delta=0$ if the centers coincide. Otherwise, $\delta$ equals 1 plus the number of edges in the shortest path connecting a vertex in the first center to a vertex in the second.

- If one of the centers consists of one vertex $u$ and the other of two vertices $v, w$, then $\delta$ equals 0.5 plus the number of edges in the shortest path connecting $u$ to $v$ or $w(\delta=0.5$ if $u=v$ or $u=w)$.

In Figure 2(b) we see that the average distance of centers in randomly generated unrooted caterpillars is extremely small, and it does not seem to depend on the number of vertices. Moreover, further experiments suggest that the distance is never greater than 1, even for large caterpillars. This supports the idea that the gravity center is a good approximation of the characteristic set and, in turn, that $\rho_{c}$ is a useful alternative to $\rho$.

Acknowledgments. The authors wish to thank the referee for giving several useful comments and suggestions that improved the final version of the paper. 
Enide Andrade was supported in part by the Portuguese Foundation for Science and Technology (FCT-Fundação para a Ciência e a Tecnologia), through CIDMA - Center for Research and Development in Mathematics and Applications, within project UID/MAT/04106/2019.

\section{References}

[1] N. Abreu, C. Justel, O. Rojo, V. Trevisan, Linear Algebra Appl. 458(2014): 429-453.

[2] E. Andrade, G. Dahl, Combinatorial Perron Values of Trees and Bottleneck Matrices, Linear and Multilinear Algebra 65 (12) (2017): 1-19.

[3] G. Dahl, Majorization and distances in trees, NETWORKS, 50 (4) (2007), 251-257.

[4] M. Fiedler, Algebraic connectivity of graphs, Czechoslovak Math. J. 23 (1973): 298-305.

[5] R. Grone, R. Merris, Algebraic connectivity of trees, Czechoslovak Math. J. 37 (1987): 660-670.

[6] R. Grone, R. Merris, Ordering trees by algebraic connectivity, Graphs and Combinatorics 6 (1990): 229-237.

[7] S. Kirkland, M. Neumann, Algebraic connectivity of weighted trees under perturbation, Linear and Multilinear Algebra 42 (1997): 187-203.

[8] S. Kirkland, M. Neumann, B. Shader, Characteristic vertices of weighted trees via Perron values, Linear and Multilinear Algebra, 40 (1996): 311325.

[9] A.W. Marshall, I. Olkin, B.C. Arnold, Inequalities: Theory of Majorization and its Applications, Springer, New York, 2011.

[10] R. Merris, Characteristic vertices of trees, Linear and Multilinear Algebra 22 (1987): 115-131.

[11] H. Minc, Nonnegative Matrices, Wiley, New York, 1988. 
[12] J.J. Molitierno, Applications of Combinatorial Matrix Theory to Laplacian Matrices of Graphs. Boca Raton: CRC Press; 2012.

[13] J.M. Steele, The Cauchy-Schwarz Master Class, Cambridge University Press, 2004. 